\title{
Acute Toxic Effects of Formalin on First Year MBBS Students during Dissection In Gross Anatomy Laboratory
}

\author{
Gousia Nisa ${ }^{1}$, Bashir Ahmad Shah ${ }^{2}$, Prof.Shaheen Shahdad ${ }^{3}$, Neelofar Jan ${ }^{4}$, \\ Sayma Samoon ${ }^{5}$, Showkat Ahmad ${ }^{6}$ \\ 1, 4, 5, 6 (Postgraduate Scholar Anatomy, GMC\& Associated Hospitals, SMHS, Srinagar India) \\ ${ }^{2}$ (Associate Professor Anatomy, GMC \& Associated Hospitals, SMHS, Srinagar, India) \\ ${ }^{3}$ (Professor\& Head Deptt Of Anatomy, GMC \& Associated Hospitals, SMHS, Srinagar, India)
}

\begin{abstract}
Formalin is used as a preservative for human cadavers. The present study was conducted on 150 first year MBBS students to find out and quantify the acute toxic effects of formalin in gross anatomy laboratory. In our study $88.1 \%$ students have reported watering of eyes and also felt that it was the first symptom to develop (74.8\%) and was the most irritating and troublesome symptom(62\%) as well. Other symptoms reported were running nose with tingling sensation(30.7\%), redness of eyes(20\%), irritation of throat $(11.2 \%)$, skin problems (10.8\%),cough(12\%), headache(6.4\%), difficulty in breathing(1.1\%) and others like lack of concentration(31.2\%).Even though formaldehyde concentrations were relatively low, medical students, instructors and cadaver related workers should wear protective devices to reduce the effects of gaseous formaldehyde exposure during gross anatomy laboratory or contact cadaver.
\end{abstract}

Keywords: Anatomy, Formalin, Laboratory, MBBS students, Toxicity,

\section{Introduction}

During an anatomy class, the evaporation of formalaldehyde from cadavers, embalming fluid, could negatively affect medical students and instructors' health ${ }^{[1]}$. Formalaldehyde is the simplest aldehyde, that can be obtained from its cyclic trimer trioxane and the polymer paraformalaldehyde ${ }^{[2]}$. Formalin $\mathrm{CH}_{2}(\mathrm{OH})_{2}$ and $\mathrm{OH}$ $\left(\mathrm{CH}_{2} \mathrm{O}\right)$ N.H is $37-50 \%$ aqueous solution of dissolved formalaldehydeCH $\mathrm{CH}_{2} \mathrm{O}$. It is widely used in medical colleges, hospitals, as preservatives, disinfectant, embalming solution and in different fields like wood and plastic industries ${ }^{[3]}$. In 1867 , the German chemist August Wilhelm von Hofmann discovered formalaldehyde ${ }^{[2,}$ 4].

Formalaldehyde solutions are used as a fixative for microscopy and histology. They are also used in embalming to disinfect and temporarily preserve human and animal remains. This is prepared by mixing the commercially available formalin solution with tap water in the proportion of $3: 1^{[2,5]}$. A cadaver is embalmed via infusion of chemical substances that include formalin (which contains formaldehyde), alcohol, glycerine, carbolic acid and dye. These substances have specific roles (e.g. preservation, denaturation, solidification of tissue protein, disinfection and maintainance of the integrity of the anatomic relations), and they are usually infused via the femoral arteries or the internal carotid arteries ${ }^{[6,7]}$.

Occupational exposure to formalaldehyde by inhalation is mainly from three types of sources: thermal or chemical decomposition of formalaldehyde based resins, formalaldehyde emission from aqueous solutions like embalming fluids and the production of formalaldehyde from the combustion of a variety of organic compounds like exhaust gases ${ }^{[2,8]}$.

Formalaldehyde enters in body by breath or when it comes in contact with your skin. It is quickly absorbed from nose and the upper part of lungs. Once absorbed, formalaldehyde is very quickly broken down. Almost every tissue in the body has ability to break down formalaldehyde. It is usually converted to a non- toxic chemical called formate, which is excreted in the urine and is converted to carbon dioxide and breathed out of the body. But formalaldehyde can be toxic, allergenic and carcinogenic ${ }^{[2]}$.

Formalaldehyde has been reported to cause acute and chronic health-related problems. The most frequent symptoms are irritation of eyes, skin and upper respiratory tract, and headache which are associated with high concentration exposure ${ }^{[9]}$. It can potentially exacerbate asthma symptoms and other respiratory illnesses while chronic exposure of formalin can cause bronchitis and pneumonia. It has also been found that when formalin is swallowed, it can result in sudden death ${ }^{[3]}$. Occupational data suggests that small but significant changes may occur in lung functions following prolonged exposure in workplace.Upper airway irritation is the most common respiratory effect reported by workers and occur over a wide range of concentrations, most frequently above 1ppm. Tolerance to this level of exposure may develop within 1-2 hours. This tolerance can permit workers remaining in an environment of gradually increasing formalaldehyde concentrations to be unaware of their increasingly hazardous exposure ${ }^{[10]}$. Though Farah et al and Molhave 
have tried to study the toxic effects of formalin, there is lack of uniformity of data and no one has defined the severity of each symptom and chronological order of occurrence.

The evaporation of formalaldehyde from cadavers in gross anatomy laboratories can produce high exposures among Ist MBBS students and instructors ${ }^{[11,12]}$. This exposure can adversely affect their health. So it is the need of time to find out the toxicity of formalin and also possible ways to reduce the toxic effects. We plan to study the acute toxic effects of formalin in first year MBBS students who are exposed to it routinely during their anatomy dissection for at least two hours every day throughout the year.

\section{Materials \& Methods}

The present study was a cross-sectional study conducted in the Department of Anatomy, Government Medical College ,Srinagar. The present study was carried out on 150 healthy subjects (Ist MBBS students) between 18-23 years of age who were routinely exposed to formalin during dissection for at least 2 hours a day and at least 3 days a week. Written informed consent was taken from all of them. Students having a history of cough, asthma, respiratory symptoms, dermatological problems or allergy, were excluded from the study. They were asked to fill up the questionnaire which included questions regarding basic demographic profile and toxic effects of formalin. They were also asked to answer which was the most troublesome symptom and which symptom was the first to develop. All the data were entered in excel sheet and further analysed by using SPSS software version11.0. Intergroup analysis was carried out by using unpaired t- test.

\section{Results}

Table shows percentage distribution of incidence of toxic effects of formalin which showed that $88.1 \%$ students had watering of eyes, followed by running nose with tingling sensation in nose $(30.7 \%)$. The other symptoms reported were redness of eyes (20\%), irritation of throat $(11.2 \%)$, cough $(12 \%)$, skin problems $(10.8 \%)$, difficulty in breathing (1.3\%), unusual tiredness / dizziness (6.2\%) and others like lack of concentration $(31.2 \%)$.

\begin{tabular}{|l|l|l|}
\hline SYMPTOM & NUMBER & PERCENTAGE(\%) \\
\hline Watering of Eyes & 132 & 88.1 \\
\hline Running Nose with tingling Sensation & 46 & 30.7 \\
\hline Redness of Eyes & 30 & 20 \\
\hline Irritation of Throat & 17 & 11.2 \\
\hline Cough & 18 & 12 \\
\hline Skin Problems & 16 & 10.8 \\
\hline Difficulty in Breathing & 2 & 1.3 \\
\hline Tiredness/Dizziness & 9 & 6.2 \\
\hline Lack of concentration & 47 & 31.2 \\
\hline
\end{tabular}

$74.8 \%$ students said that the watering of eyes was the first symptom to develop, while $62 \%$ of students also reported it to be the most troublesome symptom. Only 3\% students sought medical treatment for the toxic effects of formalin. Also, when enquired about formalin toxicity, only a few students $(5.7 \%)$ were aware of the toxic effects of formalin.

In intergroup analysis between students who used masks and gloves regularly during dissection and the students who did not, showed statistically significant difference in terms of both incidence and severity of symptoms. Besides this, there was statistically no significant difference in symptoms among students wearing spectacles and students having normal vision but students wearing contact lenses had more irritating ocular symptoms.

\section{Discussion}

In the present study we have quantified the different toxic effects of formalin in terms of incidence of occurrence, chronological order and their severity in 150 Ist MBBS students. The clinical symptoms discovered were watering through eyes, skin irritation, tingling / burning sensation in nose, running nose, irritation of throat, cough, headache, giddiness and lack of concentration. The most troublesome symptom and first to develop was watering through eyes. The second one was burning nose and running nose. We also found students who do not use mask and gloves regularly for dissection are at a high risk of developing these symptoms. These results confirmed to many studies $[1,13,16]$.

Mechanism of action of formalin toxicity: Toxicity of formalin is mainly due to formalaldehyde present in it. Formalaldehyde is a flammable, colourless and readily polymerised gas at ambient temperature and is one of the major pollutants of indoor air. The primary routes of human exposure to formalaldehyde are inhalation, eye and dermal contact. Formalaldehyde being water soluble gets dissolved in the mucosa. It then causes degenerative, inflammatory and hyperplastic changes in the mucosa of the target organ. The absorbed formalaldehyde is then converted to formate by enzymes present in erythrocytes. Formate can cause cross- 
linking of nucleic acids and amino acids causing cell death. Finally formate is oxidized to carbon dioxide and is excreted via exhalation. Sometimes binding of formalaldehyde to endogenous proteins may result in formation of neoantigens/haptens. Such neoantigens may elicit an immune response.Chronic exposure to formalaldehyde has been associated with immunological hypersensitivity as measured by elevated circulating $\operatorname{IgG}$ and IgE autoantibodies to human serum albumin. In addition, a decrease in the proportion of T- cells was observed, indicating altered immunity ${ }^{[17]}$.

Considering this issue the World Health Organisation (WHO) has developed a guideline for formalaldehyde concentration in non-occupational settings at $100 \mathrm{ppb}\left(0.1 \mathrm{mg} / \mathrm{m}^{3}\right)$ for 30 minutes. This guideline was developed to protect against sensory irritation in the general population but WHO states that it also represents an exposure level at which there is negligible risk of upper respiratory tract cancer in humans. Some do not recommend use of gloves as Environmental Health Criteria 89 of International Program of Chemical Safety states, "It must be regarded that formaldehyde fluid is not absorbed directly into tissues through the skin". So the students may be allowed in some cases to touch the cadaver, treated by formaldehyde fixative, by bare hands to understand the feel of certain organs and tissues ${ }^{[6,18]}$. As is quoted by BS Mitchell "reduction in formalaldehyde concentration is not deleterious to specimen preservation but leads to a safer working environment" ${ }^{[19]}$. As for the other alternative chemicals in place of formalaldehyde, Frolich et al in 1984 tried using phenoxyethanol as its non-toxic substitute. It proved to be impractical as the amount required was large, i.e.about 600 litres for each cadaver needing continuous emersion to prevent mould formation and fixation process taking 5 to 10 months ${ }^{[20]}$. Similarly, Glutaraldehyde is an aldehyde related to formalaldehyde, with similar fixation qualities. It would be a feasible alternative, but because of the volumes that would be required, it is prohibitively expensive. Also Reinhard Pabst in his follow-up study has advocated the use of glutaraldehyde as a good substitute for formalaldehyde ${ }^{[21]}$. Some researcher also believes that ethanol glycerine fixation with thymol conservation can be a potential alternative to formalaldehyde and phenol embalming ${ }^{[22]}$.

\section{Conclusion}

Thus, formalaldehyde present in formalin definitely has a toxic effect on various body tissues which can adversely affect the health of Ist MBBS students who during their dissection course are exposed to formalaldehyde. So proper precautions should be taken to prevent formalin toxicity. Some simple measures such as increasing airflow in the affected area by opening windows and doors, by using special local exhaust ventilators in dissection halls, by minimising direct skin contact with formalin by using protective equipment such as rubber gloves, masks and aprons, exposing only the part of the cadaver that is being dissected and periodic removal of fluid dripping collected in the body trays, will help in minimising the toxic effects of formalin. But there was not so much reduction in the symptom irritation and watering of the eyes by using the spectacles ${ }^{[23]}$.

The other probable measures which can be taken will be the use of lesser concentration of formalaldehyde and other chemicals like surgical spirit, glycerine and carbolic acid will help in maintaining a good preservation of the cadavers ${ }^{[20]}$. It has also been proved that arterial injection is the lowest exposure in the embalming operation and has the least impact on overall exposure values, so these should be practised during embalming ${ }^{[3]}$.

\section{References}

[1]. Kajorn Lakchayapakorn, Pensri Watchalayarn : Formalaldehyde exposure of medical students and instructors and clinical symptoms during gross anatomy laboratory in Thammasat University: J Med Assoc Thai 2010; 93(suppl.7):S92-S98.

[2]. Binawara BK, Rajnee, Choudary S, Mathur KC, Sharma H, Goyal K: Acute effect of Formalin on pulmonary function tests in medical students: Pak J Physiol (2010); 6(2): 8-10.

[3]. Jain SR, Nahar PS, Baig MM : Study of formalin toxicity in Ist MBBS students : International Journal Of Science And Research(IJSR) 2012; vol.1(3): 233-235.

[4]. Schwarcz L. Sanitary products. MacNair-Dorland Company; 1943.p.61.

[5]. Dixit D. Role of standardized embalming fluid in reducing the toxic effects of formalaldehyde. Indian Journal of Forensic Medicine and Toxicology, 2008; 2(1): 01-06.

[6]. Girish V.Patil, Shishirkumar, Thejeshwari, Apoorva D, Javed Sharif, C.Sheshgiri, Sushant N.K: Physical reactions of Formalin used as cadaver preservative on First year Medical students. Journal of Evidence Based Medicine and Healthcare; Vol.1, Issue 5, July2014; 279-283.

[7]. Coleman.R and Kogan I. An improved low formaldehyde embalming fluid to preserve cadavers for anatomy teaching. Journal of Anatomy, 192(Pt. 3), 1998; 443-446.

[8]. Formalaldehyde, 2- Butoxyethanol and 1- tert- Butoxypropan -2-ol, IARC Monographs on the evaluation of carcinogenic risks to humans 88, Lyon, France: International Agency for Research on Cancer,2006.p.39-325.

[9]. Cheol-Woo Kim, Jae-Seok Song, Yeon- Soon Ahn, Seung-Hyun Park, Jung-Won Park, Jae-Hoon Noh and Chein-Soo Hong: Occupational asthma due to Formalaldehyde: Yonsei Med J, vol.42, no.4 (2001).

[10]. Farah Khaliq and Praveen Tripathi: Acute effects of Formalin on pulmonary functions in gross anatomy laboratory: Indian J Physiol Pharmacol (2009); 53(1): 93-96.

[11]. Akbar-Khanzadeh F, Vaquerona MU, Akbar-Khanzadeh M, Bisesi MS: Formalaldehyde exposure, acute pulmonary response and exposure control options in a gross anatomy laboratory. Am J Ind Med 1994; 26: 61-75. 
[12]. Keil CE, Akbar-Khanzadeh F, and Koneeny KA: Characterizing formalaldehyde emission rates in a gross anatomy laboratory. Appl Occup Environ Hyg 2001; 16: 967-972.

[13]. Wantke F, Focke M, Hemmer W, Tschabitscher M, Gann M, Tappler P, et al: Formalaldehyde and phenol exposure during an anatomy dissection course- a possible source of IgE mediated sensitization? Allergy 1996; 51: 837-41.

[14]. Takahashi S, Tsuji K, Fujii K, Okazaki F, Takigawa T, Ohtsuka A, et al: Prospective study of clinical symptoms and skin test reactionsin medical students exposed to formalaldehyde gas. J Dermatol. 2007; 34: 283-9.

[15]. Mizuki M, Tsuda T: Relationship between atopic factors and physical symptoms induced by gaseous formalin exposure during an anatomy dissection course. Arerugi 2001; 50:21-8.

[16]. Kunugita N, Nakashima T, Kikuta A, Kawamoto T, Arashidani K. Exposure to formalin during an anatomy dissection course; J UOEH 2004; 26: 337-48

[17]. Thrasher JD, Wojdani A, Cheung G and Henser G. Evidence of formalaldehyde antibodies and altered cellular immunity in subjects exposed to formalaldehyde in mobile homes. Arch Environ Health 1987; 42(6): 347-50.

[18]. M.Neghab, A.Soltanzadeh, AR.Choobineh: Respiratory symptoms and functional impairments induced by occupational exposure to Formalaldehyde: Iran Occupational Health, vol.7 (2).2010.

[19]. O'Sullivan E, Mitchell BS. An improved composition for embalming fluid to preserve cadavers for anatomy teaching in United Kingdom; J Anat 1983; 182: 295-7.

[20]. Frolich KW, Andersen LM, Knutsen A, Flood PF. Phenoxyethanol as a non-toxic substitute for formalaldehydein long term preservation of human anatomical specimens for dissection and demonstration purposes. Anat Rec 1984; 208: 271-8.

[21]. Pabst R. Exposure to formalaldehyde in anatomy: An occupational health hazard? The Anatomical Record 1987; 219(2): 109-12.

[22]. Hammer N, Loffler S. Ethanol -glycerine fixation with thymol conservation: A potential alternative to formalaldehyde exposure. Anat Sci Edu 2012; 5(4): 225-33.

[23]. Raja DS, Sultana B. Potential health hazards for students exposed to formaldehyde in the gross anatomy laboratory. J Environ Health2012; 74(6): 36-40. 